\title{
Habilidades e percepções do enfermeiro frente aos cuidados paliativos
}

\author{
Nurse's Skills and Perceptions Over Palliative Care
}

Habilidades y Percepciones del Enfermero Frente a Los Cuidados Paliativos

Maria Karoline Sousa Gomes ${ }^{1 *}$, Érika Verônica Mendes de Oliveira ${ }^{1}$, Államy Danilo Moura e Silva ${ }^{1}$, Juan Victor da Silva².

\section{RESUMO}

Objetivo: Analisar os estudos disponíveis na literatura acerca das habilidades e percepções do enfermeiro frente aos cuidados paliativos. Métodos: Revisão integrativa da literatura realizada nas bases de dados Literatura Latino-Americana e do Caribe em Ciências da Saúde (LILACS), Índice Bibliográfico Espanhol de Ciências da Saúde (IBECS), Base de Dados da Enfermagem (BDENF) via Biblioteca Virtual da Saúde (BVS), PubMed/MEDLINE, da National Library of Medicine. Resultados: Os 7 estudos que compuseram essa revisão foram realizados de 2015 a 2020, sendo a maioria em 2020. Em relação a abordagem, a maioria foram estudos descritivos exploratórios e descritivos qualitativos. Os estudos encontrados foram realizados em diferentes países, incluídos em totalidades na língua inglesa. Considerações finais: Constatou-se a importância das habilidades e percepções dos enfermeiros na prestação de cuidados paliativos, mostrando que os enfermeiros desempenham um papel fundamental desses cuidados por estarem na linha de frente e tendo contato frequente com esses pacientes. No entanto, mostra-se que se necessita de mais estudos nessa área, afim de facilitar o acesso desses profissionais às pesquisas, com o intuito de melhorar a qualidade $\mathrm{e}$ assistência desses cuidados e diminuindo as barreiras que ainda existem.

Palavras-chave: Enfermeiro, Habilidade clínica, Assistência paliativa.

\begin{abstract}
Objective: Analyze the studies available in the literature about the skills and perceptions of nurses facing palliative care. Methods: Integrative review of literature carried out in the Literatura Latino-Americana e do Caribe em Ciências da Saúde (LILACS - Latin American Literature and the Caribbean in Health Sciences ), Índice Bibliográfico Espanhol de Ciências da Saúde (IBECS - Spanish Bibliographic Index of Health Sciences), Base de Dados da Enfermagem (BDENF-Nursing Database), Biblioteca Virtual da Saúde (VHL - Virtual Health Library), PubMed/MEDLINE, from the National Library of Medicine. Result: The 7 studies that composed this review were held from 2015 to 2020, with the majority in 2020. Regarding approach, most were descriptive exploratory and descriptive studies qualitative. The studies found were carried out in different countries, most of them in the English language. Considerations finals: It was found the importance of nurses skills and perceptions in providing palliative care, showing that nurses play an essential role when it comes to these cares for being on the front lines and having usual contact with these patients. However, it is shown that more studies in this area are necessary, in order to easy these workers access to research, therefore providing quality and assistance of this care and breaking the barriers that still exist.
\end{abstract}

Keywords: Nurses, Clinical skill, Palliative care.

${ }^{1}$ Centro Universitário Santo Agostinho (UNIFSA), Teresina - PI. *E-mail: mkarolinesg@gmail.com

2 Universidade Estadual do Piauí (UESPI), Floriano - PI. 


\section{RESUMEN}

Objetivo: Analizar los estudios disponibles en la literatura acerca de las habilidades y percepciones del enfermero frente a los cuidados paliativos. Métodos: Revisión integrativa de la literatura realizada en las bases de datos Literatura Latino-Americana y del Caribe en Ciencias de la Salud (LILACS), Índice Bibliográfico Español de Ciencias de la Salud (IBECS), Base de Datos de enfermería (BDENF) através de la Biblioteca Virtual de la Salud (BVS), PubMed/MEDLINE, de la National Library of Medicine. Resultado: Los 7 estudios que compusieron esta revisión fueron realizados de 2015 hasta 2020, con la mayoría en 2020. Con relación a el abordaje, la mayoría fueron estudios descriptivos exploratorios y descriptivos cualitativos. Los estudios encontrados fueron realizados en diferentes países, incluidos en totalidades en lengua inglesa. Consideracionaes finales: Se constató la importancia de las habilidades y percepciones de los enfermeros en la prestación de cuidados paliativos, mostrando que los enfermeros desempeñan un papel fundamental de estos cuidados por estar en primera línea y teniendo contacto frecuente con eses pacientes. Sin embargo, se demuestra que son necesarios más estudios en esta área, para facilitar el acceso de estos profesionales a las investigaciones, con el fin de mejorar la calidad y asistencia de estos cuidados y reduciendo las barreras que aún existen.

Palabras clave: Enfermero, Habilidad clínica, Asistencia paliativa.

\section{INTRODUÇÃO}

O termo "cuidados paliativos" foi caracterizado pela Organização Mundial da Saúde (OMS) como um cuidado que promove uma melhor qualidade de vida para os pacientes e familiares que estão em uma situação de vulnerabilidade por enfrentarem uma doença que não tem possibilidade de cura. Esses cuidados são essenciais para melhorar as condições do paciente fisicamente, psicologicamente e espiritualmente, dando assim uma assistência de melhor qualidade no fim da vida (ACADEMIA NACIONAL DE CUIDADOS PALIATIVOS, 2012).

Ademais, os cuidados paliativos passaram a ser oficialmente como prática na área da saúde, na década de 1960 no Reino Unido, tendo como pioneira a médica e também enfermeira Cicely Saunders e no mesmo ano a OMS definiu o conceito e princípios dos cuidados paliativos, que inicialmente era voltada para pacientes portadores de câncer, sendo mais tarde, em 2002 ampliado para pacientes portadores de Síndrome da Imunodeficiência Adquirida (AIDS), portadores de doenças renais, neurológicas, cardíacas e degenerativas (GOMES ALZ e OTHERO MB, 2016).

No entanto, diferente dos países desenvolvidos o Brasil não apresenta nenhuma política pública que planeje detalhadamente a forma de se desenvolver os cuidados paliativos. A Academia Nacional de Cuidados Paliativos (ANCP) destaca a importância de uma Política Nacional que oriente e norteie os cuidados paliativos no Brasil, ainda assim, também defende-se a ideia de ter mais profissionais paliativistas dentro do Ministério da Saúde, seria um passo importante para a melhoria do desenvolvimento das práticas dos cuidados paliativos de qualidade no Brasil (ANCP, 2018).

O Instituto Nacional do Câncer (INCA) publicou que para o melhor atendimento dos cuidados paliativos devem-se incluir investigações necessárias e eficientes para o manejo da dor, seus sinais e sintomas que estão relacionados com o tratamento e evolução da doença. Diante dos sintomas físicos, psicológicos e emocionais que crescem constantemente no paciente terminal, é necessário a execução de condutas terapêuticas de forma dinâmica, sendo voltadas para a melhora dos sintomas do paciente e levando em conta os seus limites diante da impossibilidade de cura (MINISTÉRIO DA SAÚDE, 2021).

Nesse sentido observa-se a importância dos cuidados paliativos com o passar dos anos, sendo cada vez mais evidente ter como modelo a assistência que auxilie nos processos de adoecimento e no fim da vida. Este trabalho, orienta-se na atuação do enfermeiro na assistência paliativa, suas habilidades e percepções 
frente a esses cuidados. Tendo em vista que atualmente a cronicidade de doenças com prognóstico agudo tem tido maior incidência vê-se, portanto, a extrema importância do desenvolvimento do conhecimento dos Cuidados Paliativos (FRANCO HCP, et al., 2017).

A Organização Mundial da Saúde (OMS) divulgou dados sobre a quantidade de pessoas que necessitam desses cuidados paliativos todos os anos ao redor do mundo. Concluiu-se ainda que, de 40 milhões de pessoas que necessitam desses cuidados apenas $14 \%$ recebem os cuidados, tratamentos e atendimento adequado. Nesse sentido, ressalta-se a importância de se inserir os cuidados paliativos precocemente, para que os profissionais da saúde tenham mais práticas e se façam-se especialistas nesse tipo de cuidado e assim os pacientes também se beneficiem (WORLD HEALTH ORGANIZATION, 2018).

Nesse aspecto, uma das partes imprescindível nesse processo, é a participação da família, essa deve ser acolhida e ouvida pelo enfermeiro e pela equipe, tendo a comunicação como o principal pilar, onde exige, respeito, confiança e dialogo, visando um plano terapêutico acordado por ambas as partes, se tornando eficiente e oferecendo melhor tratamento para o paciente/família (ANDRADE CG, et al., 2013).

Desta forma, observa-se a necessidade da captação dos profissionais de saúde, especialmente dos enfermeiros que saibam lidar com pacientes e famílias que estão prestes a enfrentar um momento delicado, que é a morte. Este estudo teve como objetivo analisar as evidencias acerca dos cuidados de enfermagem a pacientes em condições de cuidados paliativos.

\section{MÉTODOS}

Realizou-se uma revisão integrativa da literatura, onde foram seguidas etapas para a composição do estudo: definição do tema e elaboração da questão norteadora; investigação na literatura e controle de inclusão de estudos; agrupamento dos estudos; consideração/análise de estudos; apreciação dos resultados e exposição da revisão integrativa (MENDES KDS, et al., 2019).

A questão de pesquisa foi elaborada de acordo com a estratégia População Interesse Contexto (PICo). Sendo assim, foi considerada a seguinte estrutura para a pesquisa: P- Enfermeiros; I- Aptidão/Habilidades e Percepção; Co- Cuidados Paliativos. Dessa forma, elaborou-se a seguinte questão: Quais as evidências científicas disponíveis na literatura sobre as habilidades e percepções demonstradas por enfermeiros diante dos cuidados paliativos?

A pesquisa começou a ser desenvolvida em julho e agosto de 2021, e foram encontrados a partir de levantamentos realizados de forma online por acesso direto ao website nas seguintes base de dados: Literatura Latino-Americana e do Caribe em Ciências da Saúde (LILACS), Índice Bibliográfico Espanhol de Ciências da Saúde (IBECS), Base de Dados da Enfermagem (BDENF) via Biblioteca Virtual da Saúde (BVS), PubMed/MEDLINE, da National Library of Medicine.

Foram selecionados artigos publicados na íntegra on-line que se tratam de habilidades e percepções do enfermeiro frente aos cuidados paliativos, publicados, nos idiomas português, inglês ou espanhol. Foram excluídos artigos que não estão dentro do período da pesquisa, que não seguem os descritores e objeto de estudo e as línguas selecionadas, não incluiu: editoriais, teses, dissertações e revisões de literatura e os que não corresponderam a questão da pesquisa. Os artigos duplicados foram estabelecidos apenas uma única vez.

Foram utilizados os descritores controlados do Banco de Descritores em Ciências da Saúde (DeCS), Medical Subject Headings (MeSH). Para amplificar as buscas dos dados utilizou-se palavras chaves e sinônimos no idioma inglês, português e espanhol e por meio de leituras prévias sobre a temática de interesse. Para sistematizar a coleta da amostra, utilizou-se formulários na busca avançada levando em consideração as individualidades de cada base de dados. Os descritores foram combinados entre si através dos operadores booleanos "AND" e "OR". Optou-se por diferentes estratégias de busca tendo em vista que as bases de dados têm suas particularidades e características distintas. A síntese de busca em cada base de dados encontra-se descritas no Quadro 1. 
Quadro 1 - Etapas para elaboração da estratégia de busca adaptado de Araújo WCO (2020).

\begin{tabular}{|c|c|c|c|}
\hline $\begin{array}{l}\text { Base LILACS, BDENF e } \\
\text { IBECS via BVS }\end{array}$ & $\mathbf{P}$ & $\mathbf{I}$ & Co \\
\hline Extração & Enfermeiros & $\begin{array}{l}\text { Aptidão/Habilidades e } \\
\text { Percepção }\end{array}$ & Cuidados Paliativos \\
\hline Conversão & Enfermeiros & $\begin{array}{l}\text { Aptidão OR Competência } \\
\text { Clínica OR Percepção }\end{array}$ & $\begin{array}{l}\text { Cuidados Paliativos OR } \\
\text { Cuidados Paliativos na } \\
\text { Terminalidade da Vida }\end{array}$ \\
\hline Combinação & Enfermeiro OR Papéis dos Enfermeiros OR Enfermeiros Clínicos & $\begin{array}{l}\text { Habilidade OR Habilidade } \\
\text { Clínica } \\
\end{array}$ & $\begin{array}{l}\text { Assistência Paliativa OR } \\
\text { Assistência Paliativa }\end{array}$ \\
\hline Construção & $\begin{array}{c}\text { (mh:(Enfermeiros)) OR (Enfermeiro OR Papéis dos Enfermeiros OR } \\
\text { Enfermeiros Clínicos) }\end{array}$ & $\begin{array}{l}\text { (mh:(Aptidão OR } \\
\text { Competência Clínica OR } \\
\text { Percepção)) OR (Habilidade } \\
\text { OR Habilidade Clínica) }\end{array}$ & $\begin{array}{l}\text { (mh:(Cuidados Paliativos OR } \\
\text { Cuidados Paliativos na } \\
\text { Terminalidade da Vida)) OR } \\
\text { (Assistência Paliativa OR } \\
\text { Assistência Paliativa) }\end{array}$ \\
\hline Uso & \multicolumn{3}{|c|}{$\begin{array}{c}\text { ((mh:(Enfermeiros)) OR (Enfermeiro OR Papéis dos Enfermeiros OR Enfermeiros Clínicos)) AND ((mh:(Aptidão OR Competência Clínica } \\
\text { OR Percepção)) OR (Habilidade OR Habilidade Clínica)) AND ((mh:(Cuidados Paliativos OR Cuidados Paliativos na Terminalidade da } \\
\text { Vida)) OR (Assistência Paliativa OR Assistência Paliativa)) }\end{array}$} \\
\hline Base PubMed/MEDLINE & $\mathbf{P}$ & $\mathbf{I}$ & Co \\
\hline Extração & Enfermeiros & $\begin{array}{l}\text { Aptidão/Habilidades e } \\
\text { Percepção }\end{array}$ & Cuidados Paliativos \\
\hline Conversão & Nurses, Male & $\begin{array}{c}\text { Aptitude OR Clinical } \\
\text { Competence OR Perception }\end{array}$ & $\begin{array}{l}\text { Palliative Care OR Hospice } \\
\text { Care }\end{array}$ \\
\hline Combinação & Nurses, Male OR Nurses Role OR Nurse Clinicians & Habilidad OR Clinical Skill & $\begin{array}{l}\text { Palliative treatment OR } \\
\text { Palliative Care Nursing }\end{array}$ \\
\hline Construção & (Nurses, Male) OR (Nurses, Male OR Nurses Role OR Nurse Clinicians) & $\begin{array}{l}\text { (Aptitude OR Clinical } \\
\text { Competence OR Perception) } \\
\text { (Aptitude OR Clinical } \\
\text { Competence OR Perception) }\end{array}$ & $\begin{array}{l}\text { (Palliative Care OR Hospice } \\
\text { Care) OR (Palliative treatment } \\
\text { OR Palliative Care Nursing) }\end{array}$ \\
\hline Uso & \multicolumn{3}{|c|}{$\begin{array}{c}\text { (((Nurses, Male) OR (Nurses, Male OR Nurses Role OR Nurse Clinicians)) AND ((Aptitude OR Clinical Competence OR Perception) } \\
\text { (Aptitude OR Clinical Competence OR Perception))) AND ((Palliative Care OR Hospice Care) OR (Palliative treatment OR Palliative Care } \\
\text { Nursing) })\end{array}$} \\
\hline
\end{tabular}

Fonte: Gomes MKS, et al., 2021. Nursing)) 
Foram encontrados 785 artigos na busca nas bases de dados e que após a aplicação dos critérios de inclusão e exclusão, obteve-se uma amostra final de 9 artigos, no qual foram lidos e analisados metodologicamente. A seleção natural pode ser observada pelo fluxograma detalhado na Figura 1.

Figura 1 - Fluxograma de identificação, seleção e inclusão dos artigos, para o desenvolvimento da

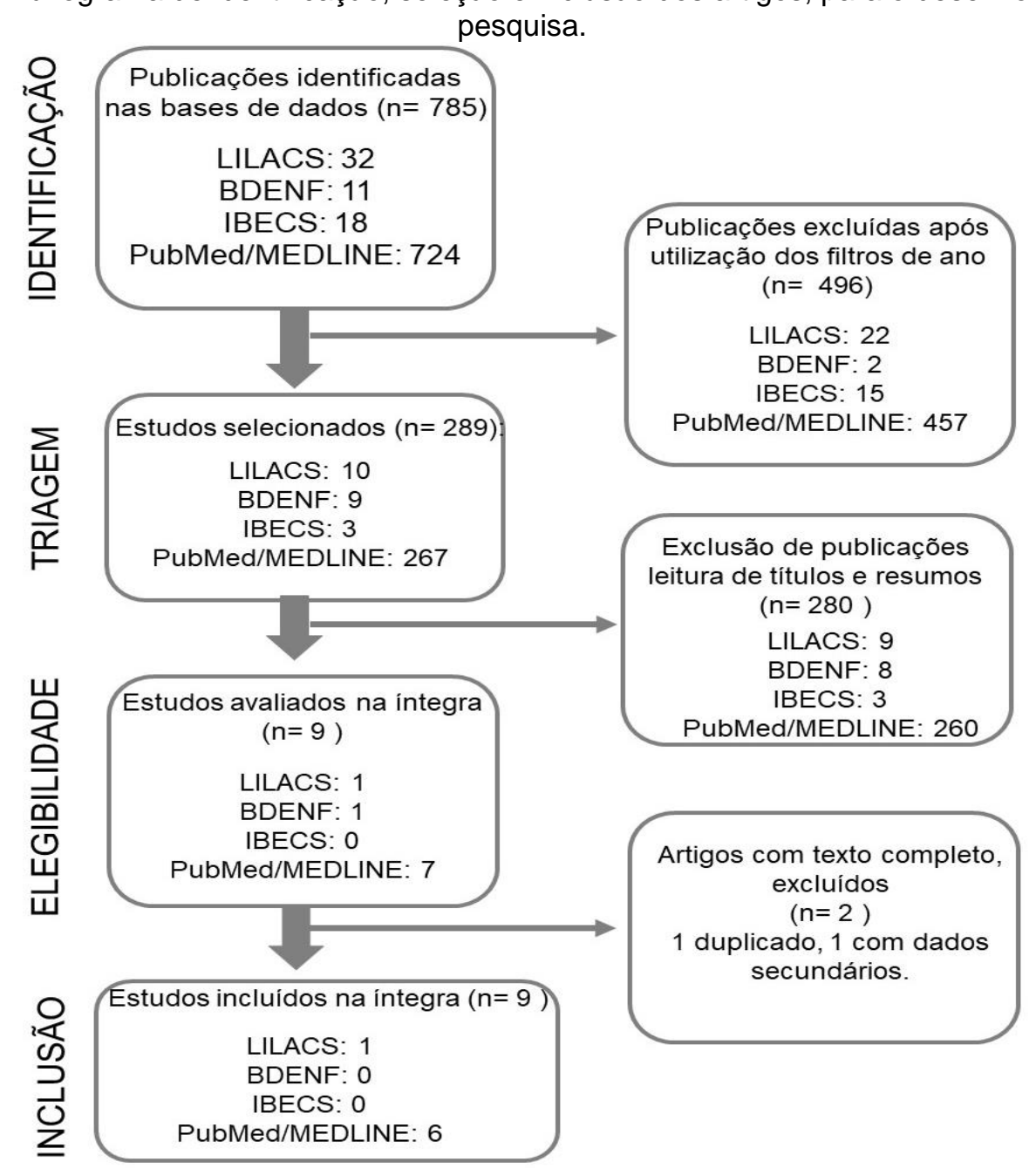

Fonte: Gomes MKS, et al., 2021.

Os dados foram analisados e extraídos por dois pesquisadores independentes, de forma simultânea onde leram e revisaram os artigos, no qual precisaram padronizar a sequência de utilização de descritores e dos cruzamentos em cada base de dados, onde em seguida precisaram comparar os dados obtidos.

\section{RESULTADOS E DISCUSSÃO}

Os resultados são apresentados no Quadro 2 de acordo com as informações sobre autor, ano, objetivo, delineamento do estudo, principais resultados e conclusões. Os 7 estudos que compuseram essa revisão foram realizados de 2015 a 2020, sendo 1 (14,3\%) em 2018, 2 (28,6\%) em 2019, 4 (57,1\%) em 2020. Em relação a abordagem, $3(42,9 \%)$ foram estudos descritivos exploratórios, $3(42,9 \%)$ descritivos qualitativos e $1(14,3)$ quase experimental.

Os estudos encontrados foram realizados em diferentes países, dos quais $1(14,3)$ nos Estados Unidos, $1(14,3 \%)$ no Brasil, $1(14,3 \%)$ na China, $1(14,3 \%)$ na Finlândia, $1(14,3 \%)$ na indonésia, $1(14,3 \%)$ no Irã e $1(14,3 \%)$ na Malásia. Todos os oito (100\%) artigos tinham sido publicados em periódicos da área de enfermagem, incluídos em totalidades na língua inglesa. 
Quadro 2 - Síntese dos estudos primários incluídos na pesquisa.

\begin{tabular}{|c|c|c|c|c|}
\hline $\begin{array}{c}\text { Autores } \\
\text { Ano }\end{array}$ & Objetivo & $\begin{array}{l}\text { Delineamento } \\
\text { do Estudo }\end{array}$ & Principais resultados & Conclusões \\
\hline $\begin{array}{l}\text { Hussin EOD, } \\
\text { et al. } \\
\text { (2018). }\end{array}$ & $\begin{array}{l}\text { Examinar os fatores associados } \\
\text { às percepções dos enfermeiros } \\
\text { sobre a qualidade dos cuidados } \\
\text { no final da vida. }\end{array}$ & $\begin{array}{l}\text { Descritivo } \\
\text { Exploratório }\end{array}$ & $\begin{array}{l}\text { Os escores médios para o conhecimento dos } \\
\text { enfermeiros sobre os cuidados no final da vida } \\
\text { e a percepção da qualidade dos cuidados no } \\
\text { final da vida foram baixos. }\end{array}$ & $\begin{array}{l}\text { Os resultados deste estudo sugerem que é } \\
\text { necessário aumentar o nível de conhecimento } \\
\text { dos enfermeiros e melhorar a sua atitude em } \\
\text { relação aos cuidados de final de vida, a fim de } \\
\text { melhorar a qualidade dos cuidados prestados } \\
\text { aos doentes terminais. }\end{array}$ \\
\hline $\begin{array}{l}\text { Dehghani F, et } \\
\text { al. } \\
\text { (2020). }\end{array}$ & $\begin{array}{l}\text { Determinar o efeito dos cuidados } \\
\text { paliativos na percepção da } \\
\text { eficácia dos enfermeiros. }\end{array}$ & $\begin{array}{c}\text { Quase } \\
\text { experimental }\end{array}$ & $\begin{array}{l}\text { Mostraram que a percepção de autoeficácia, } \\
\text { suporte psicossocial e controle dos sintomas } \\
\text { melhoram significativamente após a } \\
\text { intervenção. }\end{array}$ & $\begin{array}{l}\text { Com base nos resultados a educação em } \\
\text { cuidados paliativos tem o potencial de } \\
\text { aumentar a percepção dos enfermeiros. Uma } \\
\text { vez que todos os membros da equipe de saúde, } \\
\text { incluindo os enfermeiros desempenham um } \\
\text { papel importante na prestação de cuidados } \\
\text { paliativos. }\end{array}$ \\
\hline $\begin{array}{l}\text { Carmen WH, } \\
\text { et al. } \\
\text { (2019). }\end{array}$ & $\begin{array}{l}\text { Avaliar a percepção dos } \\
\text { enfermeiros sobre o que constitui } \\
\text { um cuidado no fim da vida ideal } \\
\text { no hospital e avaliar as barreiras } \\
\text { percebidas pelos enfermeiros } \\
\text { para a prestação de cuidados. }\end{array}$ & $\begin{array}{l}\text { Descritivo } \\
\text { Exploratório }\end{array}$ & $\begin{array}{l}\text { Os cinco fatores mais percebidos que } \\
\text { constituem os cuidados paliativos ideal, } \\
\text { incluíram os seguintes: as famílias conhecem } \\
\text { e seguem os desejos do paciente, } \\
\text { preocupações emocionais do paciente } \\
\text { identificadas e bem administradas, pacientes } \\
\text { participando da tomada de decisões. A cinco } \\
\text { barreiras era que os médicos estão muito } \\
\text { ocupados, enfermeiras muito ocupadas, sal } \\
\text { privada com espaço insuficiente, enfermeiros } \\
\text { tem treinamento limitado e a família tem } \\
\text { expectativas irrealistas do prognóstico do } \\
\text { paciente. }\end{array}$ & $\begin{array}{l}\text { Os resultados identificaram elementos } \\
\text { essenciais para o cuidado paliativo hospitalar } \\
\text { ideal, envolvendo não apenas pacientes e } \\
\text { famílias na decisão e cuidado, mas também } \\
\text { documentação e questões ambientais no nível } \\
\text { do sistema de saúde e as necessidades de } \\
\text { fortalecimento da mão de obra e experiência no } \\
\text { nível da política de cuidados paliativos. }\end{array}$ \\
\hline
\end{tabular}




\begin{tabular}{|c|c|c|c|c|}
\hline $\begin{array}{c}\text { Autores } \\
\text { Ano }\end{array}$ & Objetivo & $\begin{array}{l}\text { Delineamento } \\
\text { do Estudo }\end{array}$ & Principais resultados & Conclusões \\
\hline $\begin{array}{c}\text { Hokka M, et al. } \\
\text { (2020). }\end{array}$ & $\begin{array}{l}\text { Sintetizar as competências de } \\
\text { enfermagem necessárias em } \\
\text { cuidados paliativos e se essas } \\
\text { competências diferem entre os } \\
\text { níveis de cuidados paliativos }\end{array}$ & $\begin{array}{l}\text { Descritiva } \\
\text { Qualitativa }\end{array}$ & $\begin{array}{l}\text { Identificaram-se seis dimensões diversas das } \\
\text { competências de liderança, comunicação, } \\
\text { nomeadamente lermagem } \\
\text { colaboração, clínica, étino-legal e psicossocial } \\
\text { e espiritual. Os relatórios raramente definiam } \\
\text { o nível de cuidados paliativos e cobriam uma } \\
\text { ampla gama de configurações de saúde. }\end{array}$ & $\begin{array}{l}\text { Os enfermeiros de uma ampla gama de } \\
\text { competências para fornecer cuidados paliativos } \\
\text { de qualidade. Poucos estudos enfocaram quais } \\
\text { competências são relevantes para um nível } \\
\text { específico de cuidados paliativos. }\end{array}$ \\
\hline $\begin{array}{l}\text { Kurnia TA, et } \\
\text { al. } \\
\text { (2020). }\end{array}$ & $\begin{array}{l}\text { Analisar a relação entre o } \\
\text { conhecimento e a autoconfiança } \\
\text { dos enfermeiros na aplicação de } \\
\text { cuidados paliativos em unidade } \\
\text { de terapia intensiva. }\end{array}$ & $\begin{array}{c}\text { Descritivo } \\
\text { Exploratório }\end{array}$ & $\begin{array}{l}\text { Os resultados da análise univariada } \\
\text { mostraram que a maioria dos entrevistados } \\
\text { apresentou alta na autoconfiança, mas } \\
\text { possuíam menos conhecimento relacionado } \\
\text { aos cuidados paliativos na UTI. }\end{array}$ & $\begin{array}{l}\text { São necessárias sessões de familiarização e } \\
\text { treinamentos relacionados a isso, enfocando as } \\
\text { crenças do enfermeiro em suas habilidades. }\end{array}$ \\
\hline $\begin{array}{c}\text { Harrington KJ, } \\
\text { et al. (2019). }\end{array}$ & $\begin{array}{l}\text { Promover o envolvimento } \\
\text { emocional para melhorar as } \\
\text { percepções e atitudes da equipe } \\
\text { sobre o cuidado de pacientes no } \\
\text { final da vida ou próximos a ele. }\end{array}$ & $\begin{array}{l}\text { Descritivo } \\
\text { Qualitativo }\end{array}$ & $\begin{array}{l}\text { As evidências demonstram que o } \\
\text { envolvimento emocional e a resiliência entre } \\
\text { os profissionais de saúde estão } \\
\text { correlacionados com melhores resultados de } \\
\text { qualidade e inversamente, que o esgotamento } \\
\text { e o estresse afetam negativamente a } \\
\text { segurança do paciente. }\end{array}$ & $\begin{array}{l}\text { Refletiu apreço pelas discussões em pequenos } \\
\text { grupos e pelas oportunidades de conversar } \\
\text { com colegas fora da unidade. Observou-se o } \\
\text { valor do envolvimento emocional os esforços } \\
\text { educacionais para melhorar os cuidados de } \\
\text { enfermagem no final da vida. }\end{array}$ \\
\hline $\begin{array}{l}\text { Silveira NB, et } \\
\text { al. } \\
\text { (2020). }\end{array}$ & $\begin{array}{l}\text { Buscou-se conhecer a } \\
\text { percepção dos pacientes acerca } \\
\text { dos procedimentos terapêuticos } \\
\text { utilizados pelos trabalhadores de } \\
\text { enfermagem para o alivio da } \\
\text { dor. }\end{array}$ & $\begin{array}{l}\text { Descritivo } \\
\text { Qualitativo. }\end{array}$ & $\begin{array}{l}\text { Mediante a análise os dados foram } \\
\text { organizados em três categorias: significado e } \\
\text { limitações causadas pela dor: expressão de } \\
\text { pacientes: procedimentos terapêuticos } \\
\text { praticados pela equipe de enfermagem para } \\
\text { avaliar a dor e instrumentos para avaliação da } \\
\text { dor. }\end{array}$ & $\begin{array}{l}\text { Para promover o alivio da dor, necessita-se } \\
\text { compreender seu significado para os pacientes, } \\
\text { considerando as dimensões físicas e } \\
\text { emocionais que a envolvem. }\end{array}$ \\
\hline
\end{tabular}

Fonte: Gomes MKS, et al., 2021. 
Os artigos incluídos na presente revisão integrativa variam conforme o desenho da metodologia, da população/amostra e das limitações das pesquisas. Nesse contexto, os resultados devem ser abordados com cautela, pois podem envolver casos específicos, não podendo ser generalizados.

Os Cuidados Paliativos (CP) são essenciais para a contribuição dos cuidados no fim da vida e a sua prática se foca no paciente, desvinculando-se assim da prática curativista onde se prioriza a melhora e a cura do paciente, nos CP o foco é no alivio da dor e seus impactos. Ainda assim, existem fatores que acabam por influenciar a prática: como a falta de preparação profissional que são necessários para a realização dos $\mathrm{CP}$ (COUTO DS, 2020).

Apesar desses cuidados estarem sendo executados com êxito ainda se vê a sua expansão ocorrendo de forma vagarosa. Ainda é preciso ter uma análise mais detalhada acerca da implementação desses cuidados, visando ter melhores promoções para suas execuções, tendo em vista que, mesmo com a sofisticação dos hospitais brasileiros, ainda é possível ver que ao se tratar desses pacientes ainda falta preparação para lidar com eles e seus familiares (MACÊDO JALJ, 2015).

Esta revisão de literatura revelou as percepções dos enfermeiros aos cuidados paliativos, demonstrando assim a importância da equipe de enfermagem nessa área, com o objetivo de melhorar as técnicas e abordagens dos cuidados paliativos, visto que é a categoria profissional que mais tem contato com os pacientes que necessitam de CP.

\section{Percepções do enfermeiro}

Os pacientes de cuidados paliativos apresentam sintomas comuns como fadiga, ansiedade, dificuldade de concentração, depressão, náuseas, dores, padrão regular de sono prejudicado, entre outros. E esses sintomas podem ser percebidos pelo profissional de enfermagem na linha de frente ao CP, pois estão em frequente contato com esses pacientes. Por isso necessita de buscas de conhecimento, para oferecer um tratamento baseado em evidência (SANTOS RS, et al., 2020).

Nesse sentido, a enfermagem é fundamental no tratamento dos CP quando se trata no manejo da dor, visto que é formado por uma equipe com profissionais com diversas competências técnicas, pautadas no princípio do conforto do paciente. Os enfermeiros têm como principal objeto de trabalho a prescrição de cuidados, sendo assim primordial o mesmo estar atento às necessidades do paciente. Por vez, vale ressaltar a importância da enfermagem na atuação do controle e manejo da dor (RODRIGUES JLR, et al., 2020).

Ademais, um questionário estruturado transversal foi conduzido com 553 enfermeiras que trabalhavam em um hospital universitário na Malásia e constatou-se que os enfermeiros ainda têm bastante dificuldade na aplicação dos cuidados paliativos, mostrando assim, necessário aumentar o nível de conhecimentos dos enfermeiros. Evidenciou-se também que muitos desses profissionais não buscavam aprimorar seus conhecimentos acerca dos $\mathrm{CP}$, principalmente em ralação ao manejo da dor e sintomas quando disponibilizados treinamentos para o enriquecimento das práticas. Sendo assim, mostra-se que a falta de conhecimento aliada a displicência dos profissionais de enfermagem, acarretam uma desprimorosa aplicação dos cuidados (HUSSIN EOD, et al., 2018).

Outrossim, um estudo com amostragem randomizada realizado no Irã onde foram incluídos 40 indivíduos, mostra que embora os cuidados paliativos seja o que os pacientes e suas famílias precisam e merecem, muitos enfermeiros não são aptos para prestar este tipo de serviço, precisando assim de treinamento adequado para que a qualidade de vida dos pacientes e suas famílias não sejam prejudicadas. $O$ treinamento adequado do profissional da enfermagem vai possibilitar um cuidado de alta qualidade, oferecendo uma boa assistência que é essencial nesse momento para o paciente e sua família (DEHGHANI F, et al., 2020; KURNIA TA, et al., 2020).

Tendo em vista que o cuidado é a singularidade da profissão de enfermagem. Neste sentido, os enfermeiros estão envolvidos diretamente na prestação de cuidados paliativos, portanto sua produtividade é de fundamental importância e um dos principais fatores que influenciam estes cuidados é a sua auto eficácia, sendo assim fundamental e determinante para uma boa atuação nesses cuidados. O conhecimento é um constituinte imprescindível para a boa atuação da enfermagem, melhora o desempenho levando assim a conduta ideal (DEHGHAN F, et al., 2020). 
Cuidar de pacientes terminais nos hospitais é comum. No entanto, pouco se sabe sobre os elementos importantes e as barreiras para o atendimento de pacientes em situação de cuidados paliativos ideais da perspectiva da equipe, o que é crucial para o atendimento nesses cuidados de qualidade em ambientes hospitalares. A assistência sublime para esses pacientes que se encontram no fim da vida não se resume apenas ao fornecimento do alivio das dores e sintomas, a boa comunicação com o paciente e família também é fundamental nos cuidados de qualidade, tendo em vista que os pacientes tem preocupações emocionais, medos e inseguranças, é válido levar em consideração essas questões para um bom planejamento da assistência prestada levando em conta a boa resposta as situações emocionais desses pacientes (CARMEN WH, et al.,2019).

Além disso, uma das habilidades que o enfermeiro tem que ter é em relação ao alívio das dores, para se promover o alivio é necessário que o enfermeiro saiba o que isso significa para os pacientes, considerando assim as dimensões físicas e emocionais que envolvem cada paciente. A dor é um dos cuidados fundamentais da enfermagem, tendo em vista que os enfermeiros passam mais tempo com os seus pacientes, eles conhecem os sinais de dor e buscam agir no alívio da mesma para medidas de conforto e isso é imprescindível nas práticas dos cuidados (SILVEIRA NB, et al., 2020).

As habilidades da equipe de enfermagem devem ser voltadas para o controle e avaliação de sinais e sintomas, sendo necessária para o auxílio da equipe multiprofissional, para que assim haja estabelecimento de prioridades para cada paciente, atendendo as necessidades individuais. Por este motivo o enfermeiro tem destaque na equipe de cuidados paliativos (ACADEMIA NACIONAL DE CUIDADOS PALIATIVOS, 2012).

Por outro lado, um estudo quantitativo com abordagem transversal mostrou que um dos obstáculos e barreiras enfrentados por enfermeiros frente aos CP foi a falta de conhecimento relacionado a esses cuidados. Nesse sentido, mostrando assim necessário a necessidade de familiarização e de mais treinamentos relacionados a esses cuidados, revelando a importância do treinamento dos enfermeiros e de suas habilidades em relação aos CP (KURNIA TA, et al., 2020).

Nesse sentido, a equipe entra com a proposta de estabilização da doença, o tratamento será conduzido a uma linha de cuidados mais exclusivos, tendo como priorização o conforto do paciente. Nessa lógica, vão ser discutidos os objetivos do tratamento que foquem no alivio e nas limitações do mesmo, tendo em vista que cada paciente possui suas particularidades é importante que se leve em consideração as condutas e objetivos no plano terapêutico respeitando assim cada individualidade (BRAZ MS e FRANCO MHP, 2017).

Por certo, é necessário mostrar empatia pelo paciente, pois ao saber escutar o paciente e suas inseguranças, entender os seus sentimentos tendo uma boa comunicação, contribui para que o profissional entenda e o avalie melhor, visto que na maioria das vezes os profissionais de enfermagem acabam por levar em conta apenas a sensação fisiológica, não levando em considerações os aspectos psicológicos ligados à dor, sendo que essa envolve não apenas aspectos fisiológicos, mas também comportamentais, afetivos e o profissional deve considerar todas essas dimensões ao avaliar o paciente, avaliando-o por inteiro e não olhando apenas para um lado (SILVEIRA NB, et al., 2020).

\section{Desempenho dos enfermeiros nas práticas dos cuidados}

Os enfermeiros desempenham um papel fundamental ao atendimento dos pacientes e precisam dar um atendimento de alta qualidade, suas habilidades em fornecer cuidados no final da vida devem ser constantemente avaliadas e aprimorada, afim de sempre melhorar suas competências em relação a esses cuidados (HARRINGTON KJ, et al., 2019; KURNIA TA, et al., 2020).

Um workshop baseado em evidências realizado no Estados Unidos constatou que na educação destinada para melhorar as habilidades deve ser abordado componentes afetivos nos cuidados de enfermagem. Nesse contexto, mostra que o envolvimento emocional e humano é correlacionado com a qualidade dos resultados. Nesse sentido, é importante levar em conta atender as necessidades dos profissionais, pois o esgotamento e o estresse acabam por se tornar uma barreira para o fruto de bons resultados no cuidado paliativo de qualidade (HARRINGTON KJ, et al., 2019; DEHGHAN F, et al.,2020). 
Por outro lado, a conversa entre a equipe de enfermagem contribui para os bons resultados, as trocas de experiências e de relatos acabam por ajudar a ampliar os conhecimentos fazendo assim que se tenha uma melhora significativa nas atitudes e resultados sobre os cuidados prestados, mostrando assim que a conversa e o diálogo com colegas também é de grande ajuda e importância (HARRINGTON KJ, et al.,2019).

\section{Competências e habilidades dos enfermeiros na aplicabilidade dos cuidados paliativos}

Os enfermeiros desempenham um papel crucial nos cuidados paliativos. Portanto, precisam de um conjunto de competências e habilidades para uma boa prestação de CP. Identificou-se em um estudo efetuado na Finlândia, as dimensões e diversas competências da enfermagem como: liderança, comunicação, colaboração clínica, ético-legal e psicossocial e espiritual. Faz-se necessário, portanto, que mais pesquisas sejam efetuadas para definir as competências da enfermagem para os diferentes níveis de CP, buscando assim uma melhora na execução e prática (HOKKA M, et al., 2020).

No tratamento farmacológico a equipe de enfermagem deve ser apta para manipulação de fármacos destacando os opioides, que apresentam percentuais satisfatórios em relação ao alívio da dor, a manipulação correta dos fármacos influencia diretamente nas respostas clínicas do paciente como o tempo de internação e custo do tratamento (JÚNIOR E, et al., 2017).

A comunicação é um item muito importante para a relação entre enfermeiro e paciente, é fundamental para que o profissional da enfermagem interprete angústias, aflições e ansiedade do paciente. Essa interação deve ocorrer em um momento oportuno com clareza, sem rodeios para que o paciente e a família tenham um melhor entendimento da situação na qual estão enfrentando. A equipe tem contato direto com paciente família, sendo assim torna-se fundamental a preparação para uma boa comunicação durante a prestação de cuidados. A comunicação em situações delicadas como os cuidados paliativos é uma habilidade fundamental para os profissionais da enfermagem pois vai além de informar as condições do paciente tratamentos e prognósticos. É necessário que se entenda as angústias e o momento que a família e paciente estão passando diante da terminalidade (BELLAGUARDA R, et al., 2020).

Dessa forma, o profissional da enfermagem deve também reconhecer as emoções e a particularidade de cada família, para uma orientação e comunicação, pois este reconhecimento visa a identificação do cuidador principal e demais familiares, para que sejam evitados desequilíbrios emocionais devido a dificuldades do tratamento e a perda (ACHURY DM e PINILLA M, 2016).

\section{CONSIDERAÇÕES FINAIS}

Constatou-se com esse estudo a importância das habilidades e percepções do enfermeiro na prestação dos cuidados no fim da vida. Diante disso, percebe-se que é de suma importância a abrangência de mais estudos nesta área, a fim de melhorar as habilidades da enfermagem na qualidade da prestação desses cuidados. As evidências científicas acerca das habilidades e percepções do enfermeiro foram diversificadas, o que indica que são necessárias mais pesquisas para definir as competências da enfermagem na contribuição dos cuidados paliativos. Conclui-se, portanto, para o bom desempenho das habilidades é necessário o acesso destes profissionais a mais pesquisas, para que a atuação da assistência de enfermagem prestada aos pacientes seja fornecida da melhor forma.

\section{REFERÊNCIAS}

1. ACHURY DM, PINILLA M. La comunicación con la familia del paciente que se encuentra al final de la vida. Enferm. Univ. 2016; 13(1): 55-60.

2. ACADEMIA NACIONAL DE CUIDADOS PALIATIVOS (ANCP). Panorama de Cuidados Paliativos no Brasil. 2018. Disponível em: https://paliativo.org.br/wp-content/uploads/2018/10/Panorama-dos-Cuidados-Paliativos-no-Brasil2018.pdf. Acessado em: 05 de novembro de 2021.

3. ACADEMIA NACIONAL DE CUIDADOS PALIATIVOS (ANCP). Manual de cuidados paliativos. 2012. Disponível em: https://dms.ufpel.edu.br/static/bib/manual_de_cuidados_paliativos_ancp.pdf. Acessado em: 11 de março de 2021.

4. ANDRADE CG, et al. Cuidados paliativos: a comunicação como estratégia de cuidado para o paciente em fase terminal. Ciênc.saúde coletiva, 2013; 18(9): 2523-2530. 
5. ARAÚJO WCO. Recuperação da informação em saúde: construção, modelos e estratégias. Conv. Ciênc.Inform, 2020; 3(2): 100-134.

6. BELLAGUARDA R, et al. Simulação realística como ferramenta de ensino na comunicação de situação crítica em cuidados paliativos. Esc. Anna Nery, 2020;24(3)e20190271.

7. BRAZ MS, FRANCO MHP. Profissionais Paliativistas e suas Contribuições na Prevenção de Luto Complicado. Psicol. cienc. prof, 2017; 37(1): 90-105.

8. CHAN CWH, et al. Nurses' perceptions of and barriers to the optimal end-of-life care in hospitals: A cross-sectional study. J Clin Nurs, 2020; 29(7-8): 1209-1219.

9. DEHGHANI F, et al. Effect of palliative care training on perceived self-efficacy of the nurses. BMC Palliat Care, 2020; $4 ; 19(1): 63$.

10. FRANCO HCP, et al. Papel da enfermagem na equipe de cuidados paliativos: a humanização no processo da morte e morrer. RGS, 2017; 17(2): 48-61.

11. GOMES ALZ, OTHERO MB. Cuidados paliativos. Estud. av, 2016; 30(88): 155-166.

12. HARRINGTON KJ, et al. Improving Attitudes and Perceptions About End-of-Life Nursing on a Hospital-Based Palliative Care Unit. J Hosp Palliat Nurs, 2019; 21(4): 272-279.

13. HÖKKÄ M, et al. Nursing competencies across different levels of palliative care provision: A systematic integrative review with thematic synthesis. Palliat Med, 2020;34(7):851-870.

14. HUSSIN EOD, et al. Factors associated with nurses' perceptions about quality of end-of-life care. Int Nurs Rev, 2018; 65(2): 200-208.

15. KURNIA TA, et al. The relationship between nurses' knowledge and self-confidence in implementing palliative care in an intensive care unit. Int J Palliat Nurs, 2020; 2; 26(4): 183-190.

16. MACÊDO JALJ. Cuidados Paliativos No Brasil. Monografia (Medicina) - Universidade Federal Da Bahia Faculdade De Medicina Da Bahia, Salvador, 2015; 38 p.

17. MACHADO JC, et al. O fenômeno da conspiração do silêncio em pacientes em cuidados paliativos: uma revisão integrativa. Enfermería Actual de Costa Rica, 2019; 36: 92-103.

18. MENDES K.D.S, et al. Uso de gerenciador de referências bibliográficas na seleção dos estudos primários em revisão integrativa. Texto contexto - enferm, 2019; 28: 1-13.

19. MINISTÉRIO DA SAÚDE. Instituto Nacional de Câncer. Ações de Controle do Câncer de Colo de Útero: cuidados paliativos. Brasil, 2021. Disponível em: https://www.inca.gov.br/controle-do-cancer-do-colo-do-utero/acoes-decontrole/cuidados-paliativos. Acesso em: 5 de nov. 2021.

20. SANCHES C, et al. Desafios da assistência de enfermagem em cuidados paliativos: revisão integrativa. Enfermagem em Foco, 2020; 11(5).

21. SANTOS RS, et al. Indicadores de qualidade aplicados na assistência de enfermagem em cuidados paliativos: Revisão integrativa da literatura. Enfermagem em Foco, 2020; 11(2).

22. SILVEIRA NB, et al. Procedimentos terapêuticos de enfermagem no contexto da dor: percepção de pacientes. Enferm. Foco, 2016; 7(1): 61-65.

23. RODRIGUES JLR. Cuidados de enfermagem no manejo da dor de pacientes adultos e idosos em cuidados paliativos. Revista de Enfermagem do Centro Oeste Mineiro, 2020;10: e3680.

24. OLIVEIRA NJ, et al. O papel da enfermagem no tratamento não farmacológico da dor de pacientes oncológicos. Rev. Dor, 2017; 18(3): 261-265.

25. VASQUE TCS, et al. Percepção dos trabalhadores de enfermagem acerca do cuidado ao paciente em terminalidade no ambiente hospitalar. Texto contexto - enferm, 2016; 25(3): e0480014.

26. WORLD HEALTH ORGANIZATION (WHO). Noncommunicable diseases and their risk factors - Palliative Care. Disponivel em: https://www.who.int/home/cms-decommissioning. Acesso em: 10 de nov. de 2021. 